\title{
Exactly solvable interacting vertex models
}

\author{
Francisco C. Alcaraz ${ }^{1}$ and Matheus J. Lazo ${ }^{2}$ \\ ${ }^{1}$ Instituto de Física de São Carlos, Universidade de São Paulo, Caixa Postal 369, \\ 13560-590, São Carlos, SP, Brazil \\ E-mail: alcaraz@if.sc.usp.br \\ ${ }^{2}$ Centro Tecnológico de Alegrete, Universidade Federal de Santa Maria/Unipampa, \\ Alegrete, RS, Brazil \\ Departamento de Física, Universidade Federal de Santa Maria, 97111-900, \\ Santa Maria, RS, Brazil \\ E-mail: lazo@smail.ufsm.br
}

\begin{abstract}
.
We introduce and solve a special family of integrable interacting vertex models that generalizes the well known six-vertex model. In addition to the usual nearestneighbor interactions among the vertices, there exist extra hard-core interactions among pair of vertices at larger distances. The associated row-to-row transfer matrices are diagonalized by using the recently introduced matrix product ansatz. Similarly as the relation of the six-vertex model with the XXZ quantum chain, the row-to-row transfer matrices of these knew models are also the generating functions of an infinite set of commuting conserved charges. Among these charges we identify the integrable generalization of the XXZ chain that contains hard-core exclusion interactions among the spins. These quantum chains already appeared in the literature. The present paper explains their integrability.
\end{abstract}

Keywords: Integrable spin chains (vertex models), Solvable lattice models, Quantum integrability (Bethe Ansatz) 


\section{Introduction}

The six-vertex model was introduced by Pauling in order to explain the residual entropy of the ice at zero temperature. The model turns out to be of great interest for the physics and mathematics of many body interacting systems, due to its integrability. The rowto-row transfer matrix of the six-vertex model is the generating function for an infinite set of commuting non-trivial charges in involution [1]. The XXZ quantum chain being one of these charges. For a quantum system to be integrable its Hamiltonian should belong to an infinite set of commuting operators. The integrability of the XXZ quantum chain is then a consequence of the infinite number of commuting charges generated by the six-vertex model. For this reason the six-vertex model is considered as a paradigm of integrability in statistical mechanics [2]-[5]

The connection of vertex models and quantum chains was also observed in a great variety of general vertex models and quantum chains thanks to the development of the quantum inverse scattering method [QISM] [6]-[8]. The QISM allows the construction of the vertex models and the associated quantum chains from the solutions of the YangBaxter equations (see [9]-11] for reviews). Basically we should expect that associated to any integrable quantum chain in one dimension should exists a vertex model whose row-to-row transfer matrix is the generating function for the quantum chain.

On the other hand, along the recent years, it has been shown that it is possible to generalize several known integrable quantum chains by preserving their integrability. These generalizations are obtained through the introduction of suitable hard-core exclusion interactions. Examples of these generalizations were obtained for the XXZ quantum chain [12, 13], spin-1 Fateev-Zamolodchikov and Izergin-Korepin models [14], $S U(N)$ Sutherland and Perk-Schultz models [12, 15] and the Bariev model [16, 17]. Some of these extended quantum chains appear in the description of the stochastic dynamics of in the asymmetric exclusion problem with particles with extended sizes [18, 15], [19]-[23]. The exact solution of these quantum chains were not obtained by the QISM but directly by the coordinate Bethe ansatz [24] or by the matrix product ansatz [25]-[28]. Consequently, the two dimensional vertex model generating these quantum chains are not known, and we cannot explain their integrability, up to now. We expect that similar to the quantum chains the associated vertex models should have, beyond

the nearest neigbour interactions imposed by the lattice connectivity, also additional hard-core interactions.

Recently an interacting five-vertex model in a square lattice was introduced and solved [29]. In this model there exist hard-core interactions along the diagonal of the square lattice. The exact solution was obtained only for the eigenspectra of the diagonalto-diagonal transfer matrix, that distinctly from the row-to-row transfer matrix does not generate an infinite set of conserved charges.

In this paper we are going to introduce a family of integrable interacting vertex models that generalize the six-vertex model and explains the integrability of the XXZ quantum chains with hard-core interactions. The row-to-row transfer matrices 
associated to these vertex models generate an infinite set of conserved charges, that include the Hamiltonians of the hard-core interacting quantum chains.

In the six-vertex model the interactions are between nearest neighbour vertices and are ruled by the geometrical connectivity of the allowed vertex configurations of the lattice. The interacting energy among two vertices is zero for allowed configurations or infinite otherwise. The family of interacting vertex models we introduce in this paper have additional interactions. Besides the usual nearest-neighbour interactions, imposed by the lattice connectivity, there exist also interactions among vertices at larger distances.

The exact solution of transfer matrices associated to vertex models or quantum Hamiltonians are usually obtained through the Bethe ansatz [24] in its several formulations. The ansatz asserts that the amplitudes of the eigenfunctions of these operators are given by a sum of appropriate plane waves. Instead of making use of the Bethe ansatz, the solution we derive will be obtained through a matrix product ansatz (MPA).

Under the general name of MPA several methods were introduced in the literature along the years. The first formulation was done for the description of the ground-state eigenfunction of some special nonintegrable quantum chains, the so called valence-bond solid models [30]-33]. MPA becomes also a successfull tool for the exact calculation of the stationary probability distribution of some stochastic one dimensional systems [34][36]. An extension of this last MPA, called dynamical MPA was introduced in [37, 38] and extended in [39]. This last ansatz gives the time-dependent probability distribution for some integrable systems.

The MPA we are going to use in this paper, in order to solve the new family of integrable vertex models, was introduced in [25]-[28]. This ansatz was applied with success in the evaluation of the eigenspectra of several integrable quantum Hamiltonians [25]-[27], transfer matrices [29, 40] and the time-evolution operator of stochastic systems [28]. According to this ansatz, the amplitudes of the eigenfunctions are given in terms of a product of matrices where the matrices obey appropriated algebraic relations. In the case of the Bethe ansatz the spectral parameters and the amplitudes of the plane waves are fixed, apart from a normalization constant, by the eigenvalue equation of the Hamiltonian or transfer matrix. On the other hand, in the MPA the eigenvalue equation fixes the commutation relations of the matrices defining the ansatz.

The layout of the paper is as follows. In section 2 we introduce the interacting vertex models we are going to solve. In section 3 we present the row-to-row transfer matrices associated to the vertex models. In section 4 we explain the MPA and give the exact solution of the vertex models. In section 5 we derive the associated quantum chains that commute with the row-to-row transfer matrices, solved in section 4. Finally in section 6 we conclude the paper with a general discussion. 


\section{The interacting four-vertex model}

The family of interacting vertex models we introduce are defined on a square lattice with $\mathrm{M}$ rows and L columns and toroidal boundary conditions. At each horizontal (vertical) lattice bond we attach an arrow pointing to the left or right (up or down) direction. These arrows configurations can be equivalently described by the vertex configurations of the lattice. A vertex configuration at a given site (center) is formed by the four arrows attached to its links. Similar to the asymmetric six-vertex model [41, 42] we impose that the allowed arrow configurations only contain vertices satisfying the ice rules: two of the arrows pointing inward and the other two pointing outward of its center. For the standard six-vertex model there are six possible configurations for the vertices. These configurations are showed in Fig. 1a with their respective energies $\varepsilon_{1}, \ldots, \varepsilon_{6}$. The partition function is given by the sum of all possible vertex configuration with the Boltzmann weights given by the product of the fugacities $e^{-\beta \varepsilon_{i}}(i=1, \ldots, 6)$ of the vertices. In Fig. 1 $\mathrm{b}$, a more convenient notation is introduced, in which we only drawn, with the corresponding fugacities $a_{0}, a_{1}, b_{1}, b_{2}, c_{1}$ and $c_{2}$, the arrows pointing to the left or down of the center defining the vertex.

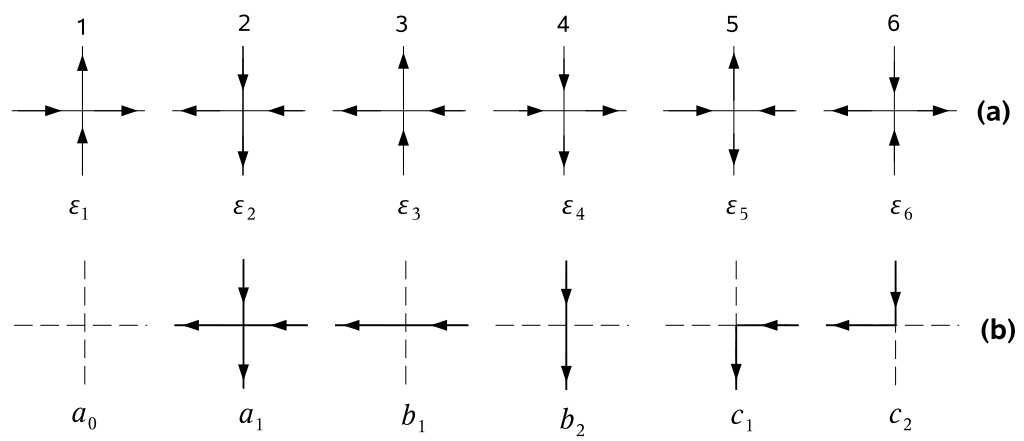

Figure 1. The six possible vertex configurations allowed by the ice rules, with their respective energies and fugacities. In (a) we draw all arrows and in (b) we give a representation where only the arrows pointing down and left are drawn.

As a consequence of the ice rules and the periodicity of the lattice the number of down arrows $n$ (or up arrows $L-n$ ) is conserved on each row of vertical bonds.

It is important to notice that the ice rules imply the existence of interactions among the nearest-neighbor vertices. These interactions have a zero or infinite value. For example (see Fig. 1b), the vertex with fugacity $a_{0}$ has an infinite interaction energy if the vertex on its right side is one of the vertices $\left(a_{1}, b_{1}, c_{2}\right)$ or the vertex on its left side is one of the vertices $\left(a_{1}, b_{1}, c_{1}\right)$. The model with no extra interactions, besides those given by the connectivity of the vertices on the lattice, is the well known six-vertex model. The partition function of the model is given by the sum of all possible vertex configurations with the Boltzmann weights given by the product of the fugacities of the vertices. The six-vertex model is exactly integrable for arbitrary values of the fugacities, and is considered a prototype of an exact solvable model [2]-[4,,41, 42]. 
We consider a special family of interacting four-vertex models. Besides the previously mentioned nearest-neighbor interactions (infinite or zero) imposed by the lattice connectivity, the models also contain interactions among pairs of vertices at larger distances. The allowed four vertex configurations, with their respective configurations are the vertices 1, 3, 5 and 6 shown in Fig. 1. Contrary to the six-vertex model the vertex configurations with fugacities $a_{1}$ and $b_{2}$ are forbidden. Such interacting fourvertex models are labeled by two fixed positive integer $s_{1}$ and $s_{2}$ that may take the values $s_{1}, s_{2}=1,2, \ldots$. These parameters specify the additional interactions among the vertices that occur when there are vertices of types $c_{1}$ and $c_{2}$ at distances equal to $s_{1}$ or $s_{2}$, in lattice units along the horizontal lines of the square lattice. A pair of vertices at distance $(l=1,2, \ldots)$, in units of lattice spacing, along this horizontal line interacts following the rulest

(a) The interaction energy is zero if one of the vertices is $a_{0}$ or $b_{1}$.

(b) If the pair of vertices is of type $c_{1}-c_{1}$ or $c_{2}-c_{2}$ the interaction energy is infinity if $l \leq s_{1}+s_{2}$.

(c) If the pair of vertices is of type $c_{1}-c_{2}\left(c_{2}-c_{1}\right)$ the interaction energy is infinity if $l<s_{1}\left(l<s_{2}\right)$ and zero if $l>s_{1}\left(l>s_{2}\right)$. In the special case where $l=s_{1}\left(l=s_{2}\right)$ the interaction energy is finite and produces, besides the fugacity of the vertices, a contribution to the Boltzmann weight $z_{1}\left(z_{2}\right)$ given by

$$
z_{1}=\frac{b_{1} b_{2}}{c_{1} c_{2}} \quad \text { and } \quad z_{2}=\frac{a_{0} a_{1}}{c_{1} c_{2}}
$$

The use of the missing fugacities $a_{1}$ and $b_{1}$ to denote the interactions $z_{1}$ and $z_{2}$ in (1) is convenient for the forthcoming analysis. In Fig. 2 we show some examples of allowed and not allowed configurations containing two arrows in the model with $s_{1}=2$ ans $s_{2}=1$.

In general, the contribution of a given pair of vertices is zero if the pair is not allowed (infinite interaction energy) or is given by the product of their fugacities (zero interaction energy). The exception to this rule happens when we have the pair $c_{1}-c_{2}$ $\left(c_{2}-c_{1}\right)$ at the distance $s_{1}\left(s_{2}\right)$ along the horizontal line (see Fig. 2). In this case, from (11), the total contribution to the Boltzmann weight is given by $c_{1} c_{2} z_{1}=b_{1} b_{2}$ $\left(c_{1} c_{2} z_{2}=a_{0} a_{1}\right)$.

We can also extend our model to the cases where the interaction parameters $s_{1}, s_{2}$ may take the value zero. In these cases the interaction follows the previous rules (a)-(c) but instead of (1) we have the generalized form

$$
z_{1}=\frac{b_{1} b_{2}}{c_{1} c_{2}} b_{1}^{-\delta_{s_{1}, 0}} \quad \text { and } \quad z_{2}=\frac{a_{0} a_{1}}{c_{1} c_{2}} a_{0}^{-\delta_{s_{2}, 0}}
$$

where $\delta_{s, 0}$ is the usual Kronecker delta. In this generalization we may have the pair of vertices $c_{1}-c_{2}\left(c_{2}-c_{1}\right)$ at distance $s_{1}=0\left(s_{2}=0\right)$. On this case the total contribution

$\ddagger$ For simplicity we denote a given vertex by its fugacity, and a pair of vertices where the vertex fugacity $c_{1}$ is on the left (right) of $c_{2}$ by $c_{1}-c_{2}\left(c_{2}-c_{1}\right)$, respectively. 


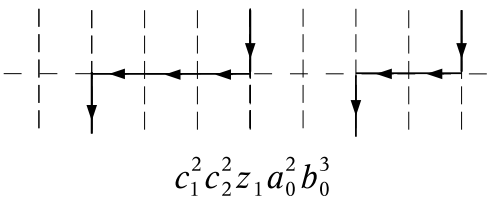

(a)
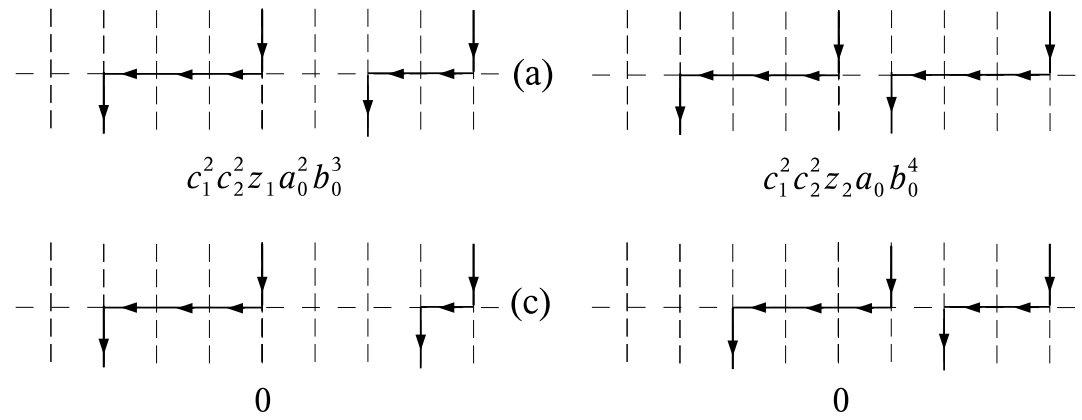

Figure 2. Configurations containing two arrows in a lattice of size $L=9$, for the model with $s_{1}=2$ and $s_{2}=1$. The configurations $(a)$ and $(b)$ are allowed and their respective Boltzmann weights contributions are shown. Configurations $(c)$ is not allowed due to the occurence of a pair $c_{1}-c_{2}$ at distance $1<s_{1}$. configuration $(d)$ is also not allowed because the two vertices $c_{2}$ are at distance $3 \leq s_{1}+s_{2}$.

comming from the pair is now $c_{1} c_{2} z_{1}=b_{2}\left(c 2 c_{1} z_{2}=a_{1}\right)$. The models where $s_{1}=0$ and $s_{2} \neq 0\left(s_{2}=0\right.$ and $\left.s_{1} \neq 0\right)$ are equivalent to interacting five vertex models where only the vertex $a_{1}\left(b_{2}\right)$ of Fig. 1 is not allowed. The interactions on these models, according to rule (a), forbid arrows at distances smaller than $s_{2}+1\left(s_{1}+1\right)$. Moreover the pair of vertices $c_{1}-c_{2}\left(c_{2}-c_{1}\right)$ interacts as in rule $(c)$. The special case where $s_{1}=s_{2}=0$ recovers the standard six-vertex model, with interactions given only by the lattice connectivity and the ice rules.

In summary, the general vertex models defined by the rules (a)-(c) and by (2), contains interacting four-vertex models $\left(s_{1} \neq 0, s_{2} \neq 0\right)$, interacting five-vertex models $\left(s_{1}=0, s_{2} \neq 0\right.$ or $\left.s_{1} \neq 0, s_{2}=0\right)$ and the standard six-vertex model $\left(s_{1}=s_{2}=0\right)$.

It is important to notice that the interactions, due to rule (b), forbid two vertical arrows at distances smaller than $s_{1}+s_{2}+1\left(s_{1}, s_{2}=0,1,2, \ldots\right)$. This can be interpreted as if the vertical arrows have an effective size $s_{1}+s_{2}+1\left(s_{1}, s_{2}=0,1,2, \ldots\right)$, in units of lattice spacing. A vertical arrow on a given link has hard-core interactions that exclude the occupation of other vertical arrows at the link itself as well as the $s_{1}$ nearest links on its left and $s_{2}$ nearest links on its right. In Fig. 3 we represent pictorically an allowed (a) and a not allowed (b) configuration of arrows for the interacting four-vertex model with $s_{1}=2$ and $s_{2}=1$. In this example the arrows have an effective size $s_{1}+s_{2}+1=4$. In the particular case $s_{1}=s_{2}=0$ the arrows have a unite size as it should be expected in the standard six-vertex model.

Similarly as the connection of the six-vertex model with the XXZ quantum chain[4] we are going to show in Sec. 3 that our interacting model, with general parameters $s_{1}$ and $s_{2}$, is also related to quantum spin chains with hard-core exclusion interactions [12, 13. The related models are the XXZ quantum chains with exclusion of up spins ( $\sigma^{z}$-basis) at distances smaller than $s_{1}+s_{2}+1$ [12, 13] and the time-evolution operator of the asymmetric exclusion problem of particles of size $s_{1}+s_{2}+1$, in units of lattice spacing [15, 28]. 

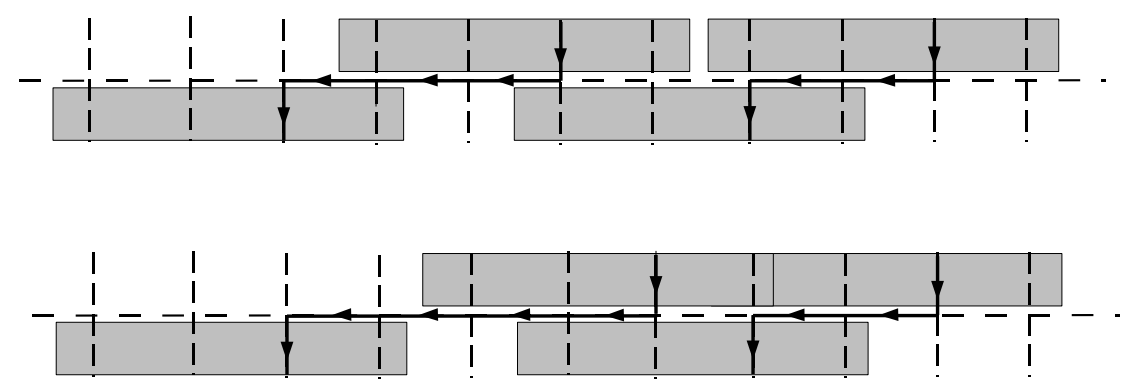

Figure 3. Examples of an allowed (a) and not allowed (b) configuration of the interacting four-vertex model with $s_{1}=2$ and $s_{2}=1$. The vertical arrows have an effective hard-core size $s=s_{1}+s_{2}+1=4$.

\section{The row-to-row transfer matrix of the interacting vertex models}

We denote a given configuration of $n$ vertical arrows located at $x_{1}, \ldots, x_{n}$ along the horizontal line of the lattice by $\left|x_{1}, \ldots, x_{n}\right\rangle$. The row-to-row transfer matrix $T_{s_{1}, s_{2}}$, with elements

$$
\left\langle x_{1}, \ldots, x_{n}\left|T_{s_{1}, s_{2}}\right| x_{1}^{\prime}, \ldots, x_{n}^{\prime}\right\rangle=a_{0}^{n_{1}} b_{1}^{n_{3}} c_{1}^{n_{5}} c_{2}^{n_{6}} z_{1}^{n_{z_{1}}} z_{2}^{n_{z_{2}}},
$$

gives the Boltzmann weight contribution to the partition function due to vertical arrow configurations $\left|x_{1}, \ldots, x_{n}\right\rangle$ and $\left|x_{1}^{\prime}, \ldots, x_{n}^{\prime}\right\rangle$ on two consecutive rows. In (3) $n_{i}$ are the numbers of vertices of type $i(i=1,3,5,6)$ and $n_{z_{1}}\left(n_{z_{2}}\right)$ is the number of pairs $c_{1}-c_{2}$ $\left(c_{2}-c_{1}\right)$ at distance of $s_{1}\left(s_{2}\right)$ units along the horizontal line (see Fig. 2). The partition function of the interacting vertex model, due to the periodic boundary condition of the lattice, is given by

$$
Z=\operatorname{Tr}\left[T_{s_{1}, s_{2}}^{M}\right]
$$

As we discussed in the last section in the cases where $s_{1}=0, s_{2} \neq 0$ and $s_{1} \neq 0, s_{2}=0$ the four-vertex models are equivalent to interacting five-vertex models. We can also verify directly that the corresponding transfer matrices $T_{0, s_{2}}$ and $T_{s_{1}, 0}$ are related to those of models with $s_{1} \neq 0$ and $s_{2} \neq 0$, namely,

$$
T_{s_{1}, s_{2}}=\left(\frac{b_{1}}{a_{0}}\right)^{n s_{1}} \mathcal{P}^{-s_{1}} T_{0, s_{1}+s_{2}}=\left(\frac{a_{0}}{b_{1}}\right)^{n s_{2}} \mathcal{P}^{s_{2}} T_{s_{1}+s_{2}, 0}
$$

where $\mathcal{P}$ is the horizontal translation operator, i. e., $\mathcal{P}\left|x_{1}, \ldots, x_{n}\right\rangle=\left|x_{1}+1, \ldots, x_{n}+1\right\rangle$. This last relation enable us to relate transfer matrices of distinct models $\left(s_{1}, s_{2}\right)$ and

$\S$ In the particular case where we have no vertical arrows $(n=0)$ or in the case where $s_{1}=s_{2}=0$ (six-vertex model) and $x_{i}=x_{i}^{\prime}(i=1, \ldots, n)$ there exist two possibilities to connect the vertical arrows. In this case Eq. (3) has two terms and should be replaced by $\left\langle x_{1}, \ldots, x_{n}\left|T_{s_{1}, s_{2}}\right| x_{1}, \ldots, x_{n}\right\rangle=$ $a_{1}^{n} b_{1}^{L-n}+b_{2} a_{0}^{L-n}$. 
$\left(s_{1}^{\prime}, s_{2}^{\prime}\right)$, provide $s_{1}+s_{2}=s_{1}^{\prime}+s_{2}^{\prime}$, i. e.,

$$
T_{s_{1}, s_{2}}=\left(\frac{b_{1}}{a_{0}}\right)^{n\left(s_{1}-s_{1}^{\prime}\right)} \mathcal{P}^{s_{1}^{\prime}-s_{1}} T_{s_{1}^{\prime}, s_{2}^{\prime}}=\left(\frac{a_{0}}{b_{1}}\right)^{n\left(s_{2}-s_{2}^{\prime}\right)} \mathcal{P}^{s_{2}-s_{2}^{\prime}} T_{s_{1}^{\prime}, s_{2}^{\prime}} .
$$

\section{The Matrix Product Ansatz for the interacting vertex models}

The transfer matrix (3) with toroidal boundary conditions has a $U(1) \times Z_{L}$ symmetry due to the conservation of vertical arrows along the horizontal lines and the translation symmetry along the horizontal direction. Consequently the associated Hilbert space can be separated into block disjoint sectors labeled by the number $n$ of vertical arrows $\left(n=0,1, \ldots,\left[L /\left(s_{1}+s_{2}+1\right)\right]\right)$ (we denote by $[x]$ the integer part of $\left.x\right)$ and the momentum eigenvalues $P\left(P=\frac{2 \pi}{L} l, l=0,1, \ldots, L-1\right)$ of the translation operator $\mathcal{P}=e^{-i \hat{P}}$. We want to solve, in each of these sectors, the eigenvalue equation

$$
T_{s_{1}, s_{2}}\left|\Psi_{n, P}\right\rangle=\Lambda_{n}\left|\Psi_{n, P}\right\rangle
$$

where $\Lambda_{n}$ and $\left|\Psi_{n, P}\right\rangle$ are the eigenvalues and eigenvectors of $T_{s_{1}, s_{2}}$, respectively. Theses eigenvectors can be written in general as

$$
\left|\Psi_{n, P}\right\rangle=\sum_{x_{1}, \ldots, x_{n}}^{*} \mathcal{A}\left(x_{1}, \ldots, x_{n}\right)\left|x_{1}, \ldots, x_{n}\right\rangle,
$$

where $\mathcal{A}\left(x_{1}, \ldots, x_{n}\right)$ is the amplitude corresponding to the arrows configuration with $n$ vertical arrows located at sites $\left(x_{1}, \ldots, x_{n}\right)$, respectively. The symbol $(*)$ in the sum means the restriction to the configurations where $x_{i+1}-x_{i}>s_{1}+s_{2}(i=1, \ldots, n-1)$, $x_{1} \geq 1, s_{1}-s_{2}<x_{n}-x_{1}<L-\left(s_{1}+s_{2}\right)$. Since $\left|\Psi_{n, P}\right\rangle$ is also an eigenvector with momentum $P$, then the amplitudes should satisfy

$$
\frac{\mathcal{A}\left(x_{1}, \ldots, x_{n}\right)}{\mathcal{A}\left(x_{1}+1, \ldots, x_{n}+1\right)}=e^{-i P}
$$

for $x_{n}<L$, while for $x_{n}=L$ we have

$$
\frac{\mathcal{A}\left(x_{1}, \ldots, L\right)}{\mathcal{A}\left(1, x_{1}+1, \ldots, x_{n-1}+1\right)}=e^{-i P} \text {. }
$$

The exact solution of the transfer matrix (3) is obtained by an appropriate ansatz for the unknown amplitudes $\mathcal{A}\left(x_{1}, \ldots, x_{n}\right)$. As shown in the last section our model reduces to the standard asymmetric six-vertex model in the case where $s_{1}=s_{2}=0$. In this particular case the model is known to be exact solvable through the Bethe ansatz [2, 41, 42] and also by the MPA [40] introduced in [25]-[27]. In the present paper we are going to derive the exact solution of the interacting vertex models with $s_{1}, s_{2}=0,1, \ldots$ by using the PMA we propose in [27]. This ansatz asserts that to any amplitude in (8) there exists a one-to-one correspondence with an ordered matrix product

$$
\mathcal{A}\left(x_{1}, \ldots, x_{n}\right) \Longleftrightarrow E^{x_{1}-1} A E^{x_{2}-x_{1}-1} A \cdots E^{x_{n}-x_{n-1}-1} A E^{L-x_{n}},
$$

where the matrices $A$ and $E$ are associated with the sites where we have a vertical arrow or not, respectively. Actually the objects $A$ and $E$ are not necessarily matrices 
but abstract operators with an associative product $\amalg$. Their commutation relations will be fixed by imposing the validity of the eigenvalue equation of the transfer matrix (7). A well-defined eigenfunction is obtained, apart from a normalization factor, if all the amplitudes are uniquely related. Equivalently, in the subset of words (products of matrices) of the algebra containing $n$ matrices $A$ and $(L-n)$ matrices $E$, should exist only a single independent word. The relation between any two words gives a $c$ number that is equal to the ratio between the corresponding amplitudes in (11).

Since the eigenfunctions $\left|\Psi_{n, P}\right\rangle$ (8) have a well-defined momentum $P=\frac{2 \pi}{L} j$ $(j=0, \ldots, L-1)$, the relations (9) and (10) imply the following constraints for the matrix products appearing in the ansatz (11)

$$
\begin{aligned}
& E^{x_{1}-1} A E^{x_{2}-x_{1}-1} A \cdots E^{x_{n}-x_{n-1}-1} A E^{L-x_{n}}= \\
& \quad \mathrm{e}^{-i P} E^{x_{1}} A E^{x_{2}-x_{1}-1} A \cdots E^{x_{n}-x_{n-1}-1} A E^{L-x_{n}-1},
\end{aligned}
$$

for $x_{n}<L$, and for $x_{n}=L$

$$
\begin{aligned}
& E^{x_{1}-1} A E^{x_{2}-x_{1}-1} A \cdots E^{x_{n}-x_{n-1}-1} A= \\
& \quad \mathrm{e}^{-i P} A E^{x_{1}} A E^{x_{2}-x_{1}-1} A \cdots E^{x_{n}-x_{n-1}-1} A E^{L-x_{n-1}-1} .
\end{aligned}
$$

In order to proceed, in the usual way, we are going to consider first the eigensectors of $T_{s_{1}, s_{2}}$ with small values of $n$.

The case $\mathbf{n}=\mathbf{0}$.

In this case the solution of the eigenvalue equation (7) is trivial since we do not have vertical arrows between two successive rows. There are only two possible horizontal arrangements: either all bonds have a horizontal arrow or all of them are empty. In this case the vertices are all of type 1 or type 3 (see figure 1 ) and consequently the eigenvalue is given by

$$
\Lambda_{0}=a_{0}^{L}+b_{1}^{L} .
$$

\section{The case $\mathbf{n}=1$.}

We have in this case just one vertical arrow between two rows. The transfer matrix links a vertical solid line at position $x(x=1, \ldots, L)$ above a row to a vertical line at any position $y\left(L \geq x \geq y+s_{1}\right.$ or $\left.y-s_{2} \geq x \geq 1\right)$ under this row. The elements of the transfer matrix $T_{s_{1}, s_{2}}(y, x)$ in this sector with momentum $P$ are given by (3). They are given by the product of the Boltzmann weights of the vertex appearing on the row. The vertex configuration at the sites $x$ and $y$ will be of types $c_{2}$ and $c_{1}$. If the position of the line $x$ is greater or equal (less or equal) than $y$ and all the others vertices will be of types $b_{1}\left(a_{0}\right)$ and $a_{0}\left(b_{1}\right)$ depending on whether the vertices are between the positions $x$ and $y$, or not, respectively. However in the special cases where $x=y+s_{1}$ or $x=y-s_{2}$ we have also to include the fugacities $z_{1}\left(z_{2}\right)$ due to the long range interaction (2) between these vertices. Consequently the eigenvalue equation (17) for the transfer matrix (3) associated

|| In the original formulation [25, 26] of the ansatz an unnecessary additional matrix [43] and a trace operation was taken in the right hand side of (11). The MPA in (11) is formulated as in the general formulation given in [27]. 
with the components of $\left|\psi_{n, P}\right\rangle$ (11) with $n=1$ and momentum $P$, give us the algebraic relations for the matrix products

$$
\begin{aligned}
& \Lambda_{1} E^{x-1} A E^{L-x}=\sum_{y=1}^{x-s_{1}-1} a_{0}^{L-x+y-1} b_{1}^{x-y-1} c_{1} c_{2} E^{y-1} A E^{L-y} \\
& +\sum_{y=x+s_{2}+1}^{L} a_{0}^{y-x-1} b_{1}^{L-y+x-1} c_{1} c_{2} E^{y-1} A E^{L-y} \\
& +a_{0}^{L-s_{1}-1} b_{1}^{s_{1}} b_{2} E^{x-s_{1}-1} A E^{L-x+s_{1}}+b_{1}^{L-s_{2}-1} a_{0}^{s_{2}} a_{1} E^{x+s_{2}-1} A E^{L-x-s_{2}},
\end{aligned}
$$

where we inserted (2) in the last two terms in the right hand side.

The equation (15) is simplified by expressing all the matrix products in terms of a single one. This is done by imposing that the matrix $A$ depend on a spectral parameter $k$. Without loss of generality, the matrix $A$ can be written in terms of the matrix $E$ and a spectral parameter dependent matrix $A_{k}$ 䟭

$$
A=E^{-s_{1}} A_{k} E^{1-s_{2}},
$$

with $A_{k}$ satisfying the following algebraic relation with the matrix $E$

$$
E A_{k}=\mathrm{e}^{i k} A_{k} E \text {. }
$$

Inserting (16) in (15) and using (17) we can factorize (15):

$$
\begin{aligned}
& \left\{\Lambda_{1}-\sum_{y=1}^{x-s_{1}-1} a_{0}^{L-x+y-1} b_{1}^{x-y-1} c_{1} c_{2} e^{i k(y-x)}-\sum_{y=x+s_{2}+1}^{L} a_{0}^{y-x-1} b_{1}^{L-y+x-1}\right. \\
& \times c_{1} c_{2} \mathrm{e}^{i k(y-x)}-a_{0}^{L-s_{1}-1} b_{1}^{s_{1}} b_{2} \mathrm{e}^{-i k s_{1}} \\
& \left.-b_{1}^{L-s_{2}-1} a_{0}^{s_{2}} a_{1} \mathrm{e}^{i k s_{2}}\right\} E^{x-s_{1}-1} A_{k} E^{L-x+1-s_{2}}=0 .
\end{aligned}
$$

In order to produce a nonzero norm state we should impose $E^{x-s_{1}-1} A_{k} E^{L-x+1-s_{2}} \neq 0$, for $x=1, \ldots, L$. As a consequence we must have, by evaluating the sums in (18),

$$
\begin{gathered}
\Lambda_{1}=a_{0}^{L} L(k)\left(\frac{b_{1}}{a_{0}}\right)^{s_{1}} \mathrm{e}^{-i k s_{1}}+b_{1}^{L} M(k)\left(\frac{a_{0}}{b_{1}}\right)^{s_{2}} \mathrm{e}^{i k s_{2}} \\
+a_{0}^{L} \frac{c_{1} c_{2}}{b_{1}}\left(\frac{b_{1}}{a_{0}}\right)^{x} \frac{\mathrm{e}^{i k(1-x)}}{b_{1}-a_{0} \mathrm{e}^{i k}}\left(1-\mathrm{e}^{i k L}\right),
\end{gathered}
$$

where

$$
L(k)=\frac{a_{0} b_{2}+\left(c_{1} c_{2}-b_{1} b_{2}\right) \mathrm{e}^{-i k}}{a_{0}^{2}-a_{0} b_{1} \mathrm{e}^{-i k}}, \quad M(k)=\frac{a_{0} a_{1}-c_{1} c_{2}-a_{1} b_{1} i \mathrm{e}^{-i k}}{a_{0} b_{1}-b_{1}^{2} \mathrm{e}^{-i k}} .
$$

In order to satisfy (15), the eigenvalue $\Lambda_{1}$ in (19) should independ on the vertical line position $x$. Thus the last term in the right hand side of (19) must vanish. The only way to cancel this term, for non zero Boltzmann weights, is obtained by imposing $e^{i k L}=1$, that fixes the spectral parameter $k$

$$
k=\frac{2 \pi}{L} j \quad(j=0,1, \ldots, L-1) .
$$

व The most general relation $A=\sum_{j=1}^{n} A^{\alpha} A_{k_{j}} E^{\beta}$ could be used. However (16) is more convenient since otherwise the structure constants $S\left(k_{j}, k_{l}\right)$ that will appear in (32) will depend on the parameters $s_{1}$ and $s_{2}$. 
The eigenvalue (19) is then given by

$$
\Lambda_{1}=a_{0}^{L} L(k)\left(\frac{b_{1}}{a_{0}}\right)^{s_{1}} \mathrm{e}^{-i k s_{1}}+b_{1}^{L} M(k)\left(\frac{a_{0}}{b_{1}}\right)^{s_{2}} \mathrm{e}^{i k s_{2}},
$$

where the values of the spectral parameter $k$ are given by (21).

Finally, by inserting (16) and using (17) in the equations (12) and (13) we verify that $k$ coincides with the momentum of the eigenstate:

$$
k=P=\frac{2 \pi}{L} j \quad(j=0,1, \ldots, L-1) .
$$

\section{The case $\mathbf{n}=2$.}

In this sector there are two vertical arrows in the row. We have in general two types of relations for the amplitudes (11). Relations where at least one of the vertical arrows $\left(y_{1}, y_{2}\right)$ are at distance $s_{1}$ or $s_{2}$ from $\left(x_{1}, x_{2}\right)$, and those where $y_{1}$ and $y_{2}$ interlace with $x_{1}$ and $x_{2}$ : $\left(1 \leq y_{1}<x_{1}-s_{1} ; x_{1}+s_{2}<y_{2}<x_{2}-s_{1}\right)$ and $\left(x_{1}+s_{2}<y_{1}<x_{2}-s_{1}\right.$; $\left.x_{2}+s_{2}<y_{2} \leq L\right)$. Then, the eigenvalue equation (7) imply

$$
\begin{aligned}
& \Lambda_{2} E^{x_{1}-1} A E^{x_{2}-x_{1}-1} A E^{L-x_{2}}=\sum_{y_{1}=1}^{x_{1}-s_{1}} \sum_{y_{2}=x_{1}+s_{2}}^{x_{2}-s_{1}} a_{0}^{L-\left(x_{2}-y_{1}+1\right)} c_{2} \\
& \times g\left(x_{1}, y_{1}\right) f\left(y_{2}, x_{1}\right) g\left(x_{2}, y_{2}\right) E^{y_{1}-1} A E^{y_{2}-y_{1}-1} A E^{L-y_{2}} \\
& +\sum_{y_{1}=x_{1}+s_{2}}^{x_{2}-s_{1}} \sum_{y_{2}=x_{2}+s_{2}}^{L *} b_{1}^{L-\left(y_{2}-x_{1}+1\right)} c_{1} f\left(y_{1}, x_{1}\right) \\
& \times g\left(x_{2}, y_{1}\right) f\left(y_{2}, x_{2}\right) E^{y_{1}-1} A E^{y_{2}-y_{1}-1} A E^{L-y_{2}},
\end{aligned}
$$

where the symbol $*$ in the sums means that terms with $\left|y_{2}-y_{1}\right| \leq s_{1}+s_{2}$ are excluded and

$$
\begin{aligned}
& f(y, x)=\left\{\begin{array}{ll}
c_{2} a_{0}^{y-x-1} & \text { if } y>x+s_{2} \\
a_{0}^{s_{2}} a_{1} / c_{1} & \text { if } y=x+s_{2}
\end{array},\right. \\
& g(x, y)=\left\{\begin{array}{ll}
b_{1}^{x-y-1} c_{1} & \text { if } x>y+s_{1} \\
b_{1}^{s_{1}} b_{2} / c_{2} & \text { if } x=y+s_{1}
\end{array} .\right.
\end{aligned}
$$

The relation (24) connects configurations where the arrangements of vertical arrows $\left(x_{1}, x_{2}\right)$ above the row do not have the same distance of the vertical arrows $\left(y_{1}, y_{2}\right)$ bellow the row. In other words, the distance of the incoming arrows $y_{2}-y_{1}$ are in general different of the outcoming distance $x_{2}-x_{1}$. To solve the relation (24) we need now to use a generalization of the algebraic relation (16) for the case of two arrows. The generalization of (16) is done by writing the matrix $A$ in terms of two new spectral parameter matrices as:

$$
A=\sum_{j=1}^{2} E^{-s_{1}} A_{k_{j}} E^{1-s_{2}},
$$

with the commutation relations

$$
E A_{k_{j}}=\mathrm{e}^{i k_{j}} A_{k_{j}} E \quad(j=1,2),
$$

where the spectral parameters $k_{1}$ and $k_{2}$ are up to now unknown complex numbers. 
Exactly solvable interacting vertex models

Inserting (27) in (24) and using (28) and (20) we obtain, after similar manipulation as we did in the case $n=1$, the following constraint

$$
\begin{aligned}
& \sum_{j, l=1}^{2}\left[\Lambda_{2}-a_{0}^{L}\left(\frac{b_{1}}{a_{0}}\right)^{2 s_{1}} \mathrm{e}^{-i\left(k_{j}+k_{l}\right) s_{1}} L\left(k_{j}\right) L\left(k_{l}\right)-b_{1}^{L}\left(\frac{a_{0}}{b_{1}}\right)^{2 s_{2}} \mathrm{e}^{i\left(k_{j}+k_{l}\right) s_{2}}\right. \\
& \left.\times M\left(k_{j}\right) M\left(k_{l}\right)\right] \mathrm{e}^{i k_{j} s_{1}} \mathrm{e}^{-i k_{l} s_{2}} \mathrm{e}^{i k_{j} x_{1}} \mathrm{e}^{i k_{l} x_{2}} A_{k_{j}} A_{k_{l}} \\
& -\sum_{j, l=1}^{2} a_{0}^{L}\left[L\left(k_{l}\right) M\left(k_{j}\right)-\frac{a_{1} b_{2}}{a_{0} b_{1}}\right]\left(\frac{b_{1}}{a_{0}}\right)^{s_{1}-s_{2}}\left(\frac{b_{1}}{a_{0}}\right)^{x_{2}-x_{1}} \mathrm{e}^{i\left(k_{j}+k_{l}\right)\left(s_{1}-s_{2}\right)} \\
& \times \mathrm{e}^{i\left(k_{j}+k_{l}\right) x_{1}} A_{k_{j}} A_{k_{l}}-\sum_{j, l=1}^{2} b_{1}^{L}\left[L\left(k_{l}\right) M\left(k_{j}\right)-\frac{a_{1} b_{2}}{a_{0} b_{1}}\right]\left(\frac{b_{1}}{a_{0}}\right)^{s_{1}-s_{2}}\left(\frac{b_{1}}{a_{0}}\right)^{x_{1}-x_{2}} \\
& \times \mathrm{e}^{i\left(k_{j}+k_{l}\right)\left(s_{1}-s_{2}\right)} \mathrm{e}^{i\left(k_{j}+k_{l}\right) x_{2}} A_{k_{j}} A_{k_{l}} \\
& +\sum_{j, l=1}^{2} a_{0}^{L} \frac{c_{1}^{2} c_{2}^{2}\left[\mathrm{e}^{i L k_{l}} \mathrm{e}^{i k_{j}\left(s_{2}+s_{1}\right)} \mathrm{e}^{-i k_{l} s_{2}} \mathrm{e}^{i k_{j} x_{1}}-\mathrm{e}^{i k_{j} s_{1}} \mathrm{e}^{i k_{l} x_{1}}\right]}{b_{1}^{2}\left(a_{0}-b_{1} \mathrm{e}^{-i k_{j}}\right)\left(a_{0}-b_{1} \mathrm{e}^{-i k_{l}}\right)} \\
& \times\left(\frac{b_{1}}{a_{0}}\right)^{-s_{2}}\left(\frac{b_{1}}{a_{0}}\right)^{x_{2}} A_{k_{j}} A_{k_{l}} \\
& -\sum_{j, l=1}^{2} a_{0}^{L}\left[\frac{c_{1}^{2} c_{2}^{2}\left[\mathrm{e}^{i L k_{l}} \mathrm{e}^{-i k_{l} s_{2}} \mathrm{e}^{-i k_{j}} \mathrm{e}^{i k_{j} x_{2}}-\mathrm{e}^{i k_{j} s_{1}} \mathrm{e}^{-i k_{l}\left(s_{2}+s_{1}\right)} \mathrm{e}^{-i k_{l}} \mathrm{e}^{i k_{l} x_{2}}\right]}{a_{0} b_{1}\left(a_{0}-b_{1} \mathrm{e}^{-i k_{j}}\right)\left(a_{0}-b_{1} \mathrm{e}^{-i k_{l}}\right)}\right] \\
& \times\left(\frac{b_{1}}{a_{0}}\right)^{s_{1}}\left(\frac{b_{1}}{a_{0}}\right)^{x_{1}} A_{k_{j}} A_{k_{l}} \\
& +\sum_{j, l=1}^{2} a_{0}^{L} c_{1} c_{2} a_{1}\left[\frac{\mathrm{e}^{i L k_{l}} \mathrm{e}^{i k_{j}\left(s_{2}+s_{1}\right)} \mathrm{e}^{-i k_{l} s_{2}} \mathrm{e}^{i k_{j} x_{1}}}{b_{1}^{2}\left(a_{0}-b_{1} \mathrm{e}^{-i k_{l}}\right)}-\frac{\mathrm{e}^{i k_{j} s_{1}} \mathrm{e}^{i k_{l} x_{1}}}{b_{1}^{2}\left(a_{0}-b_{1} e^{-i k_{j}}\right)}\right] \\
& \times\left(\frac{b_{1}}{a_{0}}\right)^{-s_{2}}\left(\frac{b_{1}}{a_{0}}\right)^{x_{2}} A_{k_{j}} A_{k_{l}} \\
& +\sum_{j, l=1}^{2} a_{0}^{L} c_{1} c_{2} b_{2}\left[\frac{\mathrm{e}^{i L k_{l}} \mathrm{e}^{-i k_{l} s_{2}} \mathrm{e}^{i k_{j} x_{2}}}{a_{0} b_{1}\left(a_{0}-b_{1} \mathrm{e}^{-i k_{l}}\right)}-\frac{\mathrm{e}^{i k_{j} s_{1}} \mathrm{e}^{-i k_{l}\left(s_{2}+s_{1}\right)} \mathrm{e}^{i k_{l} x_{2}}}{a_{0} b_{1}\left(a_{0}-b_{1} \mathrm{e}^{-i k_{j}}\right)}\right] \\
& \times\left(\frac{b_{1}}{a_{0}}\right)^{s_{1}}\left(\frac{b_{1}}{a_{0}}\right)^{x_{1}} A_{k_{j}} A_{k_{l}}=0,
\end{aligned}
$$

where $1 \leq x_{1}<x_{2} \leq L$ with $\left|x_{2}-x_{1}\right| \geq s_{1}+s_{2}+1$. This can only be satisfied if each sum is identically zero. Moreover since $\Lambda_{2}$ should be independent of $x_{1}$ or $x_{2}$ a possible solution of (29) is obtained by imposing

$$
\begin{aligned}
\Lambda_{2}= & a_{0}^{L}\left(\frac{b_{1}}{a_{0}}\right)^{2 s_{1}} \mathrm{e}^{-i\left(k_{1}+k_{2}\right) s_{1}} L\left(k_{1}\right) L\left(k_{2}\right)+b_{1}^{L}\left(\frac{a_{0}}{b_{1}}\right)^{2 s_{2}} \mathrm{e}^{i\left(k_{1}+k_{2}\right) s_{2}} \\
& \times M\left(k_{1}\right) M\left(k_{2}\right) .
\end{aligned}
$$

The algebraic relation between the matrices $A_{k_{1}}$ and $A_{k_{2}}$ are obtained by imposing that both the second and third terms in (29) are zero independently, i. e.,

$$
A_{k_{j}} A_{k_{l}}=S\left(k_{j}, k_{l}\right) A_{k_{l}} A_{k_{j}}, \quad\left(A_{k_{j}}\right)^{2}=0 \quad(j \neq l=1,2),
$$


where

$$
S\left(k_{j}, k_{l}\right)=-\frac{L\left(k_{j}\right) M\left(k_{l}\right)-\frac{a_{1} b_{2}}{a_{0} b_{1}}}{L\left(k_{l}\right) M\left(k_{j}\right)-\frac{a_{1} b_{2}}{a_{0} b_{1}}},
$$

with $L(k)$ and $M(k)$ given by (20). Note that the structure constants $S\left(k_{j}, k_{l}\right)$ is independent of $s_{1}$ and $s_{2}$ due to the choice (27) (see the footnote related to Eq. (16)). Finally, the vanishing of the last four terms in (29) will give us relations that fix the spectral parameters values $k_{1}$ and $k_{2}$. These equations are obtained by exploring the algebraic relations (31)

$$
\mathrm{e}^{i L k_{j}}=\left(\frac{\mathrm{e}^{i k_{j}}}{\mathrm{e}^{i k_{l}}}\right)^{s_{1}+s_{2}} S\left(k_{j}, k_{l}\right) \quad(l, j=1,2 \text { and } l \neq j) .
$$

The eigenvalues are obtained by inserting the solutions $\left(k_{1}, k_{2}\right)$ of these last equations in (30). The momentum $P$ is obtained by using (27) and (28) in (12) and (13): $P=k_{1}+k_{2}$. The amplitudes of the corresponding eigenvectors are obtained from the algebraic relations (27), (28) and (31).

\section{The case of general $n$.}

The general case follows straightforwardly from the $n=2$ case. The previous calculation can be extended for arbitrary values of the number $n$ of vertical arrows. The eigenvalue equation for the transfer matrix connects the amplitudes $\mathcal{A}\left(x_{1}, \ldots, x_{n}\right)$ and $\mathcal{A}\left(y_{1}, \ldots, y_{n}\right)$ where: $1 \leq y_{1} \leq x_{1}-s_{1}, x_{1}+s_{2} \leq y_{2} \leq x_{2}-s_{1}, \ldots, x_{n-1}+s_{2} \leq y_{n} \leq x_{n}-s_{1}$ and $x_{1}+s_{2} \leq y_{1} \leq x_{2}-s_{1}, x_{2}+s_{2} \leq y_{2} \leq x_{3}-s_{1}, \ldots, x_{n}-s_{2} \leq y_{n} \leq L$. To solve the eigenvalue equation we need to extend the definition (27) and the commutation relations (28) for general $n$, i. e.,

$$
A=\sum_{j=1}^{n} E^{-s_{1}} A_{k_{j}} E^{1-s_{2}}
$$

with

$$
E A_{k_{j}}=\mathrm{e}^{i k_{j}} A_{k_{j}} E \quad(j=1, \ldots, n) .
$$

The parameters $k_{j}(j=1, \ldots, n)$ are in general unknown complex numbers that will be fixed by the eigenvalue equation (7). Inserting (34) in (7) and using the commutation relations (35) we obtain, similarly as done in the case $n=2$, the algebraic relations among the matrices $A_{k_{j}}(j=1, \ldots, n)$ :

$$
A_{k_{j}} A_{k_{l}}=S\left(k_{j}, k_{l}\right) A_{k_{l}} A_{k_{j}} \quad\left(A_{k_{j}}\right)^{2}=0 \quad(j \neq l=1, \ldots, n),
$$

where $S\left(k_{j}, k_{l}\right)$ is given by (32) and the spectral parameters $k_{j}(j=1, \ldots, n)$ are fixed by the equation

$$
e^{i L k_{j}}=-\prod_{l=1}^{n}\left(\frac{\mathrm{e}^{i k_{j}}}{\mathrm{e}^{i k_{l}}}\right)^{s_{1}+s_{2}} S\left(k_{j}, k_{l}\right) \quad(j=1, \ldots, n) .
$$

The acceptable set $\left\{k_{j}\right\}$ of spectral parameters defining the eigenvectors $\left|\Psi_{n, P}\right\rangle$ is given by the solutions of (37) where $k_{l} \neq k_{j}(j, l=1, \ldots, n)$. Since $\left(A_{k_{j}}\right)^{2}=0$, solutions of (37) with coinciding roots give us null states. 
No new algebraic relations appear for the matrices $\left\{A_{k_{j}}\right\}$ besides (35) and (36). This not easy to verify. The difficulty here is similar to that in showing the absence of many-particle scattering in the standard coordinate Bethe ansatz for vertex models [2]- [5]. The product associativity of the algebra (35) and (36) follows from the property $S\left(k_{j}, k_{l}\right) S\left(k_{l}, k_{j}\right)=1$. From the the eigenvalue equation (7) we obtain the eigenvalues

$$
\Lambda_{n}=a_{0}^{L}\left(\frac{b_{1}}{a_{0}}\right)^{n s_{1}} \prod_{j=1}^{n} \mathrm{e}^{-i k_{j} s_{1}} L\left(k_{j}\right)+b_{1}^{L}\left(\frac{a_{0}}{b_{1}}\right)^{n s_{2}} \prod_{j=1}^{n} \mathrm{e}^{i k_{j} s_{2}} M\left(k_{j}\right),
$$

where $L(k)$ and $M(k)$ are given by (20) and the spectral parameters $\left\{k_{j}\right\}$ are the solutions of (37). Finally, the momentum $P$ follows from (12), (13), (34) and (35):

$$
P=\sum_{j=1}^{n} k_{j} .
$$

Before closing this section let us give a possible representation for the matrices $E$ and $A$ of the ansatz (11) [27]. For a given solution $\left\{k_{1}, \ldots, k_{n}\right\}$ of the spectral parameter equations (37), in the sector with $n$ vertical arrows, the matrices $E$ and $\left\{A_{k_{1}}, \ldots, A_{k_{n}}\right\}$ have the following $2^{n} \times 2^{n}$ dimensional representation:

$$
\begin{aligned}
& E=\bigotimes_{l=1}^{n}\left(\begin{array}{cc}
1 & 0 \\
0 & \mathrm{e}^{-i k_{l}}
\end{array}\right) \\
& A_{k_{j}}=\left[\bigotimes_{l=1}^{j-1}\left(\begin{array}{cc}
S\left(k_{j}, k_{l}\right) & 0 \\
0 & 1
\end{array}\right)\right] \otimes\left(\begin{array}{ll}
0 & 1 \\
0 & 0
\end{array}\right) \bigotimes_{l=j+1}^{n}\left(\begin{array}{ll}
1 & 0 \\
0 & 1
\end{array}\right),
\end{aligned}
$$

where $S\left(k_{j}, k_{l}\right)$ are given by (32) and $A$ is obtained from (34). It is important to notice that the matrices $E$ and $A_{k_{j}}$ have the same functional form obtained for the formulation of the MPA for the Hamiltonian of the XXZ spin chain [27]. It happens because the matrices $E$ and $A_{k}$ satisfy the same algebraic relations (34)-(36) in both models. The only difference are the values of the spectral parameters $\left\{k_{1}, \ldots, k_{n}\right\}$ that for the present model are fixed by equation (37).

It is important to notice that all the interacting four-vertex $\left(s_{1} \neq 0, s_{2} \neq 0\right)$, fivevertex $\left(s_{1}=0, s_{2} \neq 0\right.$ and $\left.s_{1} \neq 0, s_{2}=0\right)$ and six-vertex models $\left(s_{1}=s_{2}=0\right)$ have the same functional form for the operators $A_{k_{j}}(j=1, \ldots, n)$. The only difference being the structure constants $S\left(k_{j}, k_{l}\right)$, since the spectral parameters $k_{1}, \ldots, k_{n}$ are solutions of distinct equations. As a test of our calculations we see that (37), for the case $s_{1}=s_{2}=0$ coincides with the spectral parameter equations obtained by the well known Bethe ansatz calculation of the asymmetric six-vertex model [41, 42]. Using the representation (40) and (41) we also obtain on this case the same amplitudes for the eigenfunctions as those derived by the Bethe ansatz.

We can also solve the interacting models introduced in this paper by the use of the same coordinate Bethe ansatz used in the six-vertex model [2]-[5]. In this case instead of obtaining the structure constants (32), with no dependence on $s_{1}$ or $s_{2}$, we obtain a S-matrix that depends on these parameters. Although the final spectral parameter equations (37) and the eigenfunctions for both ansatz are the same, we believe the derivation through our MPA is more elegant. 
The phase diagram of the interacting vertex models introduced in this paper can be derived from that of the six-vertex model. This follows from the spectral-parameter equations (37), that can be written as

$$
\mathrm{e}^{i\left[L-n\left(s_{1}+s_{2}\right)\right] k_{j}} \mathrm{e}^{i P\left(s_{1}+s_{2}\right)}=-\prod_{l=1}^{n} S\left(k_{j}, k_{l}\right), \quad(j=1, \ldots, n),
$$

where we have used (39). Consequently the eigenvalues belonging to the eigensector with $n$ vertical arrows and momentum $P$ of the transfer matrix $T_{s_{1}, s_{2}}$ with parameters $s_{1}$ and $s_{2}$ are related to those of the standard six-vertex model $\left(s_{1}=s_{2}=0\right)$. The related six-vertex model is defined on a cylinder with perimeter $L^{\prime}=L-n\left(s_{1}+s_{2}\right)$ and with a momentum-dependent seam spanning its length + .

\section{The interacting vertex models and generalized XXZ quantum chains}

It is well known the connections among the XXZ quantum chain and the standard asymmetric six-vertex model [4]. The row-to-row transfer matrix of the six-vertex model is the generating function of an infinite set of commuting charges. The XXZ quantum Hamiltonian being one of these charges. Since the six-vertex model is a particular case $\left(s_{1}=s_{2}=0\right)$ of our general model it is natural to expect that we can also generate generalized exact solvable XXZ quantum chains for the general case where $s_{1}, s_{2} \geq 0$.

The first step towards the derivation of those generalized quantum chains is the identification of the row-to-row transfer matrix $T_{s_{1}, s_{2}}$, for arbitrary values of $s_{1}$ and $s_{2}$, as a generating function of commuting charges.

It is important to notice that the structure constants $S\left(k_{j}, k_{l}\right)$ given in (32) do not depend on the particular values of $s_{1}$ and $s_{2}$. It only depends on the parameters $a_{0}, a_{1}$, $b_{1}, b_{2}, c_{1}$ and $c_{2}$. Consequently it is convenient to use the same parametrization used in [44] for the asymmetric six-vertex model

$$
\begin{aligned}
& a_{0}=\mathrm{e}^{-\beta \varepsilon_{1}}=\mathrm{e}^{h+\nu} \frac{\sinh (\gamma+\epsilon \mu)}{\sinh \gamma} \\
& a_{1}=\mathrm{e}^{-\beta \varepsilon_{2}}=\mathrm{e}^{-h-\nu} \frac{\sinh (\gamma+\epsilon \mu)}{\sinh \gamma} \\
& b_{1}=\mathrm{e}^{-\beta \varepsilon_{3}}=\mathrm{e}^{-h+\nu} \frac{\sinh \mu}{\sinh \gamma} \\
& b_{2}=\mathrm{e}^{-\beta \varepsilon_{4}}=\mathrm{e}^{h-\nu} \frac{\sinh \mu}{\sinh \gamma} \\
& c_{1}=\mathrm{e}^{-\beta \varepsilon_{5}}=1 \\
& c_{2}=\mathrm{e}^{-\beta \varepsilon_{6}}=1,
\end{aligned}
$$

where $\epsilon= \pm 1$ and $\gamma, \mu, h$ and $\nu$ are free parameters. The choice $c_{1}=c_{2}=1$ does not restrict the model since, due to the periodic boundary condition, these parameters

+ The phase $\mathrm{e}^{i P\left(s_{1}+s_{2}\right)}$ in the left hand side of (42) can be obtained by considering a vertex model with twisted boundaries. The twisted is obtained by introducing a seam with distinct vertex fugacities along the vertical direction. 
always appear in pairs. The symmetric interacting models are obtained by setting $h=\nu=0$.

Inserting in (32) the parameters in (43) we obtain

$$
S\left(k_{j}, k_{l}\right)=-\frac{1+\mathrm{e}^{4 h} \mathrm{e}^{i\left(k_{j}+k_{l}\right)}-\epsilon \mathrm{e}^{2 h} \cos \gamma \mathrm{e}^{i k_{l}}}{1+\mathrm{e}^{4 h} \mathrm{e}^{i\left(k_{j}+k_{l}\right)}-\epsilon \mathrm{e}^{2 h} \cos \gamma \mathrm{e}^{i k_{j}}},
$$

that shows the independence of the structure constants of the algebra defining the MPA on the parameters $\mu$ and $\nu$.

The results of previous sections imply that, for a given solution $\left\{k_{1}, \ldots, k_{n}\right\}$ of the spectral parameter equations (37), the amplitudes of the corresponding eigenfunction of $T_{s_{1}, s_{2}}$ depends only on the algebraic relations (34)-(36). Consequently all the eigenfunctions of $T_{s_{1}, s_{2}}$, for fixed values of $s_{1}$ and $s_{2}$, are the same for arbitrary values of $\mu$ and $\nu$, i. e.,

$$
\left[T_{s_{1}, s_{2}}(\mu, \nu), T_{s_{1}, s_{2}}\left(\mu^{\prime}, \nu^{\prime}\right)\right]=0 .
$$

Moreover, since from (5) $T_{s_{1}, s_{2}}$ and $T_{s_{1}^{\prime}, s_{2}^{\prime}}$ differ by the multiplication of a diagonal operator, we may write the general relation

$$
\left[T_{s_{1}, s_{2}}(\mu, \nu), T_{s_{1}^{\prime}, s_{2}^{\prime}}\left(\mu^{\prime}, \nu^{\prime}\right)\right]=0, \quad s_{1}+s_{2}=s_{1}^{\prime}+s_{2}^{\prime} .
$$

The conserved charges are derived by expanding $T_{s_{1}, s_{2}}(\mu, \nu)$ in terms of $\mu$. Since we are interested only in the derivation of the quantum chain associated to $T_{s_{1}, s_{2}}(\mu, \nu)$, in the following we are going to consider only the two leading terms in the $\mu$-expansion.

The matrix elements of $T_{s_{1}, s_{2}}(\mu, \nu)$ in the eigensector with $n$ arrows are given by $*$

$$
\begin{aligned}
& \left\langle\{y\}\left|T_{s_{1}, s_{2}}(\mu, \nu)\right|\{x\}\right\rangle=P_{s}(\{y\}) P_{s}(\{x\}) a_{0}^{L-\left(x_{n}-y_{1}+1\right)} c_{2} \\
& \quad \times g\left(x_{1}, y_{1}\right) f\left(y_{2}, x_{1}\right) g\left(x_{2}, y_{2}\right) \cdots f\left(y_{n}, x_{n-1}\right) g\left(x_{n}, y_{n}\right),
\end{aligned}
$$

if $x_{1} \geq y_{1}+s_{1}$, and

$$
\begin{array}{r}
\left\langle\{y\}\left|T_{s_{1}, s_{2}}(\mu, \nu)\right|\{x\}\right\rangle=P_{s}(\{y\}) P_{s}(\{x\}) b_{1}^{L-\left(y_{n}-x_{1}+1\right)} c_{2} \\
f\left(y_{1}, x_{1}\right) g\left(x_{2}, y_{1}\right) f\left(y_{2}, x_{2}\right) \cdots g\left(x_{n}, y_{n-1}\right) f\left(y_{n}, x_{n}\right),
\end{array}
$$

if $x_{1} \leq y_{1}-s_{2}$. In these last equations $s=s_{1}+s_{2}$ and $P_{s}(\{x\})$ projects out configurations not satisfying the hard-core exclusion constraint:

$$
P_{s}(\{x\})=\theta\left(x_{n}-x_{1}-[L-(s+1)]\right) \prod_{i=1}^{n-1} \theta\left(x_{i+1}-x_{i}-(s+1)\right),
$$

where

$$
\theta(y)=\left\{\begin{array}{lll}
1 & \text { if } \quad y \leq 0 \\
0 & \text { if } \quad y<0
\end{array}\right.
$$

is the standard step function. In (47) and (48) the functions $f(y, x)$ and $g(x, y)$ are defined in (25) and (26) and have the leading behaviour in the $\mu$-expansion

$$
\begin{aligned}
f(y, x)= & \mathrm{e}^{(h+\nu)\left(y^{\prime}-x-s_{1}-2\right)}\left\{1+\left[\left(y^{\prime}-x-s_{1}-2\right)+2 \delta_{y^{\prime}-x, s+1}\right] \epsilon \mu \operatorname{coth} \gamma\right\} \\
& \times \theta\left(y^{\prime}-x-(s+1)\right) \delta_{y, y^{\prime}-s_{1}-1}+O\left(\mu^{2}\right),
\end{aligned}
$$

* The exceptional cases considered in the footnote of page 7 should be considered separately, with no changes in the final results. 
Exactly solvable interacting vertex models

$$
\begin{aligned}
g(x, y)= & \left(\frac{\mathrm{e}^{\nu-h} \mu}{\sinh \gamma}\right)^{s_{1}}\left[\delta_{x-y^{\prime}, 0}+\frac{\mu}{\sinh \gamma}\left(\mathrm{e}^{\nu-h} \delta_{x-y^{\prime}, 1}+\mathrm{e}^{h-\nu} \delta_{x-y^{\prime},-1}\right)\right] \\
& \times \delta_{y, y^{\prime}-s_{1}-1}+O\left(\mu^{s_{1}+2}\right) .
\end{aligned}
$$

Using (51) and (52) in (47) we obtain

$$
\begin{aligned}
& \left\langle\{y\}\left|T_{s_{1}, s_{2}}\right|\{x\}\right\rangle=\left(\frac{\mathrm{e}^{\nu-h} \mu}{\sinh \gamma}\right)^{n s_{1}} \mathrm{e}^{h+\nu} P_{s}(\{y\})\left\{\prod_{i=1}^{n} \delta_{y_{i}, y_{i}^{\prime}-s_{1}-1}\right\} \\
& \times\left\{1+\frac{\mu}{\sinh \gamma} \sum_{i=1}^{L}\left[\mathrm{e}^{-2 h} \delta_{x_{i}, y_{i}^{\prime}+1}^{(L)}+\mathrm{e}^{2 h} \delta_{x_{i}, y_{i}^{\prime}-1}^{(L)}+\left(L-2 n-n s_{1}\right.\right.\right. \\
& \left.\left.\left.+2 \sum_{i=1}^{L} \delta_{x_{i+1}-x_{i}, s+1}^{(L)}\right) \epsilon \cosh \gamma\right]+O\left(\mu^{2}\right)\right\} P_{s}(\{x\}),
\end{aligned}
$$

where

$$
\delta_{x_{i+1}-x_{i}, s+1}^{(L)}=\left\{\begin{array}{cl}
\delta_{x_{i+1}-x_{i}, s+1} & \text { for } \quad i=1, \ldots, n-1 \\
\delta_{x_{n}-x_{1}, L-(s+1)} & \text { for } \quad i=n
\end{array} .\right.
$$

We can rewrite (53) in terms of spin- $\frac{1}{2}$ Pauli matrices by identifying an arrow at site $i$ as $\sigma_{i}^{z}=+1$, and we obtain

$$
\begin{aligned}
T_{s_{1}, s_{2}}(\mu, \nu)= & \mathrm{e}^{-\left[h\left(1+s_{1}\right)+\nu\right] \sum_{i=1}^{L}\left(\sigma_{i}^{z}+1\right)+L(h+\nu)}\left(\frac{\mu}{\sinh \gamma}\right)^{s_{1} \sum_{i=1}^{L} \frac{\sigma_{i}^{z}+1}{2}} \mathcal{P}^{-\left(1+s_{1}\right)} \\
& \times\left[P_{s_{1}+s_{2}}+\frac{\mu}{\sinh \gamma} H_{s_{1}, s_{2}}+O\left(\mu^{2}\right)\right]
\end{aligned}
$$

where

$$
\begin{aligned}
H_{s_{1}, s_{2}}= & P_{s_{1}+s_{2}}\left\{\sum_{i=1}^{L}\left[\epsilon_{+} \sigma_{i}^{-} \sigma_{i+1}^{+}+\epsilon_{-} \sigma_{i}^{+} \sigma_{i+1}+\frac{\Delta}{2}\left(\sigma_{i}^{z} \sigma_{i+s_{1}+s_{2}+1}^{z}+1\right)\right]\right. \\
& \left.\quad-h_{m} \sum_{i=1}^{L}\left(1+\sigma_{i}^{z}\right)\right\} P_{s_{1}+s_{2}}, \\
\epsilon_{+}= & e^{2 h}, \quad \epsilon_{-}=\mathrm{e}^{-2 h}, \quad h_{m}=\frac{s_{1}}{2}, \quad \Delta=\epsilon \cosh \gamma, \quad \epsilon= \pm 1 .
\end{aligned}
$$

In this last equation periodic boundary conditions is imposed, $\sigma_{i}^{ \pm}=\left(\sigma^{x} \pm i \sigma^{y}\right) / 2$ and $P_{s_{1}+s_{2}}$ is the diagonal operator $\left\langle\{x\}\left|P_{s_{1}+s_{2}}\right|\{x\}\right\rangle=P_{s_{1}+s_{2}}(\{x\})$ defined in (49), that projects out configurations where two up spins are distances smaller than $s_{1}+s_{2}+1$. The same expressions (55) and (56) are also obtained by inserting (51) and (52) in (48).

We see from (56) that associated to the family of solvable interacting vertex models, with interacting parameters $s_{1}$ and $s_{2}$, we have a generalized asymmetric XXZ quantum chain. This quantum chain have hard-core exclusion interactions that forbid the occupation of up spins at distances smaller than $s_{1}+s_{2}+1$. The important parameter controlling the exclusion effect is given by the combination $s=s_{1}+s_{2}$. The effect of the parameter $s_{1}$ in (56) is the same as an external magnetic field $h_{m}=\frac{s_{1}}{2}$.

The Hamiltonian (56) for the case where $s_{1}=s_{2}=0$ gives the standard asymmetric XXZ chain. The particular case where $\Delta=\epsilon_{+}+\epsilon_{-}$gives the time-evolution operator 
of the asymmetric exclusion problem where the particles have hard-core size $s_{1}+s_{2}+1$, in units of lattice spacing [18, 15].

The integrability of (56), for general values of $s_{1}$ and $s_{2}$, is already known in the literature [18, 15, 19]. What is unknown is why the inclusion of the hard-core exclusion constraints in the standard XXZ quantum chain model $\left(s_{1}=s_{2}=0\right)$, producing the Hamiltonian (56), does not destroy the integrability of the original model. This paper gives the explanation for this fact. The quantum chains (56), for a given value of $s_{1}$ and $s_{2}$, belongs to the infinite set of of commuting charges generated by the row-to-row transfer matrix $T_{s_{1}+s_{2}}$ of our interacting vertex models.

\section{Conclusions and generalizations}

We have introduced in this paper a special family of exact solvable interacting vertex models. These models are generalizations of the six-vertex model. Besides the usual nearest-neighbor interactions imposed by the lattice connectivity, the models also contains hard-core interactions along the horizontal lines of the lattice. The range of the additional interactions depends on two fixed integer parameters $s_{1}, s_{2}=0,1, \ldots$ This new family of models contain interaction four-vertex models $\left(s_{1} \neq 0, s_{2} \neq 0\right)$, interacting five-vertex models $\left(s_{1}=0, s_{2} \neq 0\right.$, or $\left.s_{1} \neq 0, s_{2}=0\right)$ and the standard six-vertex model $\left(s_{1}=s_{2}=0\right)$. These vertex models can also be interpreted as if the vertical arrows entering in the vertex configurations have an effective hard-core size $s=s_{1}+s_{2}+1$.

For a given value of $s_{1}$ and $s_{2}$ the model can be parametrized by four parameters $(\gamma, \nu, \mu$ and $h)$ (see (43) ). The exact solution of the eigenspectra of the row-to-row transfer matrix shows the independence of the eigenfunctions on the parameters $\nu$ and $\mu$, implying the commutativity of an infinite family of transfer matrices (see (45) and (46)). The expansion of the transfer matrix in terms of the free parameter $\mu$ give an infinite set of commuting conserved charges. A member of this set is a XXZ quantum chain with hard-core interactions that exclude two up spins $\left(\sigma^{z}\right.$-basis $)$ at distances smaller than $s_{1}+s_{2}+1$ [12, 13]. This quantum chain also describes the asymmetric diffusion of particles with hard-core size $s_{1}+s_{2}+1$, in units of lattice spacing [18, 15, 19]. The integrability of this quantum chain is already known [12, 13. The present paper give an explanation for this integrability, namely, the existence of an infinite set of commuting charges generated by the row-to-row transfer matrix of the introduced interacting vertex models.

The phase diagram of the introduced interacting vertex models, as discussed in Sec. 4, can be obtained from the known results of the six-vertex model. The critical phases of the models are governed by a Coulomb gas type of conformal field theories with central charge $c=1$. Since the spectral parameter equations are the same as those of the extended XXZ quantum chain [13] the compactification ratio, that fixes the critical exponents, depend on the density of vertical arrows $n$ and on the parameters $s_{1}$ and $s_{2}$.

The exact solution we obtained for the interacting vertex models introduced in this paper were done by using the matrix product ansatz introduced in [25]-[28]. The most 
elegant solution of the six-vertex model is obtained by using the $R$-matrix approach in the quantum inverse scattering method. Can we solve the introduced interacting vertex models by generalizing the $R$ matrix of the six-vertex model? Due to the appearance of nonlocal operators in the row-to-row transfer matrix we were not able to find such generalization. That is an interesting question to be answered in the future.

Similar to the hard-core generalization of the XXZ quantum chain, has been also generalized several known integrable quantum chains through the introduction of suitable hard-core exclusion interactions. As examples we have hard-core generalizations of the spin-1 Fateev-Zamolodchikov and Izergin-Korepin models [14], $S_{q} U(N)$ Sutherland and Perk-Schultz models [19, 20] and the Bariev model [11, 16]. These quantum chains with no hard-core interactions, as the standard XXZ chain, are known to be generated by the row-to-row transfer matrix of known vertex models. We believe that the same ideas presented in this paper for the generalization of the sixvertex model can also be extended to these vertex models. We expect that the vertex models generating the quantum chains with only next neighbour interactions are special cases of a larger family of integrable interacting vertex models.

\section{Acknowledgments}

This work has been partly supported by FAPESP, CNPq and CAPES (Brazilian agencies).

\section{References}

[1] Tarasov V O, Takhtajan L A and Fadeev L D, 1983 Theor. Math. Phys. 571059

[2] E. H. Lieb E H, 1967 Phys. Rev. 162162

[3] Sutherland B, Yang C N and Yang C P, 1967 Phys. Rev. Lett. 19588

[4] Baxter R J, 1982 Exactly solved models in statistical mechanics (Academic Press: London)

[5] Gaudin M, 1983 La Fonction d'Onde de Bethe (Masson: Paris)

[6] Fadeev L D and Takhtajan L A, 1981 Sov. Sci. Rev. Math. C1 107

[7] Takhtajan L A, 1983 J. Sov. Math. 232470

[8] Sklyanin E K, Takhtajan L A and Fadeev L D, 1979 Theor. Math. Phys. 40688

[9] Korepin V E, Izergin A G and Bogoliubov N M, 1992 Quantum Inverse Scattering Method, Correlation Functions and Algebraic Bethe Ansatz (Cambridge University Press: Cambridge)

[10] Essler F H L and Korepin V E, 1994 Exactly Solvable Models of Strongly Correlated Electrons (World Scientific: Singapore)

[11] Schlottmann P, 1977 Int. J. Mod. Physics B 11355

[12] Alcaraz F C and Bariev R Z, 1998 Phys. Lett. A 240247

[13] Alcaraz F C and Bariev R Z, Alcaraz F C and Bariev R Z, 1999 Statistical Physics in the Eve of the 21st Century (Series on Advances in Statistical Mechanics) vol 14, Eds. Batchelor M T and Wille L T ( World Scientific: Singapore) Preprint cond-mat/9904042 (1999).

[14] Alcaraz F C and Bariev R Z, 1999 Phys. Rev. B 593373

[15] Alcaraz F C and Bariev R Z, 1999 Phys. Rev. E 6079

[16] Yue R H and Schlottmann P, 2002 Nucl. Phys. B 647539

[17] Yue R H and Schlottmann P, 2002 Phys. Rev. B P085114

[18] Sazamoto T and Wadati M, 1999 J. Phys. Soc. Japan 662618 
[19] Alcaraz F C and Bariev R Z, 2000 Braz. J. Phys. 3013

[20] Alcaraz F C and Bariev R Z, 2000 Braz. J. Phys. 30655

[21] Shaw L B, Zia R K P and Lee K H, 2003 Phys. Rev. E 68 P021910

[22] Ferreira A A and Alcaraz R Z, 2002 Phys. Rev. E colocar P052102

[23] Schönherr G and Schütz G M, 2004 J. Phys. A: Math. Gen 378315

[24] Bethe H A, 1931 Z. Phys. 71205

[25] Alcaraz F C and Lazo M J, 2004 J. Phys. A: Math. Gen. 37 L1

[26] Alcaraz F C and Lazo M J, 2004 J. Phys. A: Math. Gen. 374149

[27] Alcaraz F C and Lazo M J, 2006 J. Phys. A: Math. Gen. 3911335

[28] Alcaraz F C and Lazo M J, 2003 Braz. J. Phys. 33533

[29] Ferreira A A and Alcaraz F C, 2006 Phys. Rev. E 74 P011115

[30] Affleck I, Kennedy T, Lieb E H and Tasaki H, 1988 Commun. Math. Phys. 115477

[31] Arovas D P, Auerbach A and Haldane F D M, 1998 Phys. Rev. Lett. 60531

[32] Fannes M, Nachtergaele B and Werner R F, 1992 Commun. Math. Phys. 144443

[33] Klumper A, Schadschneider A and Zittartz J, 1992 Z. Phys. B 87281

[34] Derrida B, Evans M R, Hakim V and Pasquier V, 1993 J. Phys. A: Math. Gen. 261493

[35] Derrida B, 1988 Phys. Rep. 30165

[36] Alcaraz F C, Dasmahapatra S and Rittenberg V 1998 J. Phys. A: Math. Gen. 31845

[37] Stinchcombe R B and Schütz G M, 1995 Phys. Rev. Lett. 75140

[38] Stinchcombe R B and Schütz G M, 1995 Europhys. Lett. 29663

[39] Popkov V, Fouladvand M E and Schütz G M, 2002 J. Phys. A: Math. Gen 357187

[40] Lazo M J, 2007 Physica A 374655

[41] Nolden I M, 1992 J. Stat. Phys. 67155

[42] Bukman D J and Shore J D, 1995 J. Stat. Phys. 781277

[43] Golinelli O and Mallick K, 2006 J. Phys. A: Math. Gen. 3910647

[44] Albertini G, Dahmen S R and Wehefritz B, 1996 J. Phys. A: Math. Gen. 29 L369 cond-mat/9509026 